\title{
February 2015 Phoenix Critical Care Journal Club: Intracranial pressure Monitoring for Fulminant Liver Failure
}

\author{
Karvellas CJ, Fix OK, Battenhouse H, Durkalski V, Sanders C, Lee WM; U S \\ Acute Liver Failure Study Group. Outcomes and complications of \\ intracranial pressure monitoring in acute liver failure: a retrospective \\ cohort study. Crit Care Med. 2014;42(5):1157-67. [CrossRef] [PubMed]
}

The fellows performed an excellent follow-up to our last journal club in which we reviewed the above article. This cohort consisted of 140 patients who underwent ICP monitoring for grade III/VI encephalopathy, and 489 non-monitored controls. Only $87 / 140(62 \%)$ of monitored patients had sufficient available data for analysis, $51 \%$ of whom had demonstrable intracranial hypertension $(\mathrm{ICH})$ that was associated with nearly a doubling of mortality (43 vs. $23 \% \mathrm{p}=0.05)$. Monitoring and control groups were dissimilar. Monitored patients were statistically more likely to require mechanical ventilation, vasopressors and dialysis, and ultimately more than twice as likely to undergo liver transplant. Reasonably, monitored patients also received more treatments aimed at reducing $\mathrm{ICH}$ including osmotic therapy and hypothermia, although no treatment protocol was stipulated. Fatal hemorrhagic complications of ICP monitoring occurred in 3 of 56 patients for whom this outcome could be determined. Overall, 21 -day mortality was similar in monitored and control patients: $33 \%$ vs. $38 \%$ $p=0.24$, but a subgroup analysis of patients with acute liver failure not due to acetaminophen showed that monitoring had an adjusted odds ratio of 3.0 $(p=0.014)$.

Karvellas' study is non-experimental, and has significant flaws, the most important of which is the lack of any specific approach to treatment of $\mathrm{ICH}$. Any hypothetical mortality benefit of ICP monitoring ought to be related to rapid and effective management once intracranial hypertension is detected. In our Critical Care service, this entails immediate bedside response by 24/7 intensivists who emergently employ a protocol that has been refined over a decade, and that takes cerebral perfusion pressure into account. We have had a long-standing belief that ICP-monitoring is beneficial in these patients and previously published our treatment protocol (1). Karvellas' study provides little information on how ICP was managed in order to reconcile their findings with ours. However it is the largest study to date, and has compelled us and our neurosurgeon associates to reconsider our long-standing practice.

Our discussions last and this month brought several pertinent issues to light through the expert input of Jeremy Payne and Doug Franz - two of our esteemed stroke-neurologists - and by extensive outside research that the fellows had the initiative to undertake, including an several literature searches and an informal survey of transplant programs around the country. The following recounting of the resulting discussions is much abbreviated.

The central issue is whether it is clinically beneficial to take immediate action if a 
patient with acute liver failure suffers a sudden rise in ICP, or a sudden drop in cerebral perfusion pressure (CPP) (2-5). Experienced clinicians still debate this issue, and there is no experimental evidence to resolve the debate. Clinicians who accept the premise that emergent action should be taken to correct abnormal ICP or CPP values will not likely be satisfied managing comatose acute liver failure patients without monitoring, or by using periodic methods to detect cerebral edema, such as brain CT scanning, transcranial doppler, or optic nerve width determination, that may lack sensitivity and fail to provide real-time detection of acute deterioration.

The fellows' survey of transplant programs around the country showed a related lack of consensus. About half of programs still used ICP monitoring in certain situations, but less so than in the past. Periodic brain CT scanning, periodic transcranial dopplers and reverse jugular bulb oxygen saturations were each used as surrogates for ICP monitoring at a minority of responding transplant centers. We determined that the current state of research and the standard of practice are such that individual programs can reasonably exercise local discretion in regards to whether and how to monitor acute liver failure patients, despite the results of Karvellas' study. Our discussion proceeded based on our non-unanimous contention that brain injury can be mitigated by emergent interventions for sudden alterations in ICP and CPP, and that the benefit of doing so outweighs the risk.

We considered multiple studies that demonstrated that non-neurosurgeons can be trained to safely place ICP monitors, but we concluded that none of us would be able to achieve the volume of procedures required to maintain competence. We considered other methods of surrogate real-time monitoring, such as jugular bulb oxygen saturation monitoring, which may be safer than ICP monitoring. But even less is known about benefits of jugular bulb oxygenation monitoring than what we know about ICP monitoring. Therefore, jugular vein oxygen saturation monitoring seems at best a topic for further research. We concluded at this point that we will invite debate with our neurosurgeons and ask for their continued support for ICP monitor placement. We might also consider a pilot trial comparing jugular vein oxygen saturation monitoring with ICP monitoring.

Robert Raschke MD, Suresh Uppalapu MD, Nick Sparacino MD and Jennifer Hall MD

Banner Good Samaritan Medical Center

Phoenix, AZ

\section{References}

1. Raschke RA, Curry SC, Rempe S, Gerkin R, Little E, Manch R, Wong M, Ramos A, Leibowitz Al. Results of a protocol for the management of patients with fulminant liver failure. Crit Care Med. 2008;36(8):2244-8. [CrossRef] [PubMed] 
2. Lee WM, Stravitz RT, Larson AM. Introduction to the revised American Association for the Study of Liver Diseases Position Paper on acute liver failure 2011. Hepatology. 2012;55(3):965-7. [CrossRef] [PubMed]

3. Siddiqui MS, Stravitz RT. Intensive care unit management of patients with liver failure. Clin Liver Dis. 2014;18(4):957-78. [CrossRef] [PubMed]

4. Lobato RD, Alen JF, Perez-Nu-ez A, Alday R, Gómez PA, Pascual B, Lagares A, Miranda P, Arrese I, Kaen A. Value of serial CT scanning and intracranial pressure monitoring for detecting new intracranial mass effect in severe head injury patients showing lesions type I-II in the initial CT scan. Neurocirugia (Astur). 2005;16(3):217-34. [CrossRef] [PubMed]

5. Behrens A, Lenfeldt N, Ambarki K, Malm J, Eklund A, Koskinen LO. Transcranial Doppler pulsatility index: not an accurate method to assess intracranial pressure. Neurosurgery. 2010;66(6):1050-7. [CrossRef] [PubMed] 